\title{
COMPARATIVE DYNAMIC ANALYSIS OF THE PORTAL CRANES OF TYPE H AND X
}

\author{
RADE VAsiLJeVić \\ Faculty of Maritime Academic Studies, Belgrade, Serbia \\ e-mail:r.r.vasiljevic@gmail.com
}

In this paper, a comparative dynamic analysis of the portal cranes of type $\mathrm{H}$ and $\mathrm{X}$ is described. The difference of the considered cranes arises from the difference of their portals. These two types of portals have the same main geometric measurements. They differ only at the medium level in the horizontal plane. The first type of the portal on the middle level has an $\mathrm{H}$-frame, while the second type has an X-frame. In this paper, dynamic parameters of the two portals have been compared. Estimation has been done to find which of the two types of portals has more favourable dynamic stability for overturn.

Keywords: portal crane, type H, type X, Finite Element Method, comparative dynamic analysis

\section{Introduction}

Portal-slewing cranes are very important from the aspect of realization of logistics processes. They are transport machinery with a boom which is connected to the portal and which has the possibility of $360^{\circ}$ slewing. The widest application has two types of the portal-slewing cranes: type $\mathrm{H}$ and type $\mathrm{X}$.

The last 50 years has seen keen interest in study on the dynamic of cranes (Abdel-Rahman et al., 2003). Dynamics of structures of the transport machinery is a fundamental topic in the structural dynamics. Dynamic analysis of portal-slewing cranes is essential from two main aspects. The first main aspect is high payload of the crane. The second main aspect is a small base of the portal in proportion to crane height. In portal-slewing cranes, there are influences which are variable in time (e.g., motion of the crane and payload swing, etc.). Payload swing contributes in a large extent to dynamic loading of cranes. The importance of the problem of payload swing was considered and confirmed in (Lee, 2004; Jerman and Haribar, 2013). The same problem is defined in standard F.E.M 1.001.

From the aspect of structural similarity of the portal cranes of type $\mathrm{H}$ and $\mathrm{X}$, it is interesting to analyse their dynamic behaviour in comparative terms. The problem of dynamic analysis of the $\mathrm{H}$ portal crane was solved in Vasiljević et al. (2016). The subject of this paper was a comparative dynamic analysis of the $\mathrm{H}$ and $\mathrm{X}$ portal-slewing cranes. It has been accepted that the two portal cranes have the same type of general boom slewing that is, they have only different types of portal construction (type $\mathrm{H}$ and type $\mathrm{X}$ ).

This paper should contribute to the knowledge of the difference of dynamic behaviour of the two portals of the $\mathrm{H}$ and $\mathrm{X}$ portal cranes excited by motion of the crane and the payload swing. The modelling of the dynamical models of the two portal types is approached by dividing the whole portal crane into two subsystems, i.e. the portal ( $\mathrm{H}$ accordingly $\mathrm{X}$ ) and the boom (identical for both portal types). The interaction between the portal and the boom is simplified by reducing the influence of payload and dead weight of the boom to corresponding points of the portal. The identical equivalent three-dimensional dynamic model of the general slewing boom 
for both crane types was formed in the first step. Three-dimensional finite element models of the $\mathrm{H}$ and $\mathrm{X}$ portals was formed in the second step. The both portals are modelled with three-dimensional linear beams. A FE technique for determining the dynamic response of structures of gantry cranes was developed by Wu et al. (2000). As in reality three-dimensional linear beams are systems with continually distributed masses, and as the three-dimensional model of the portals ( $\mathrm{H}$ and $\mathrm{X}$ ) is complex, the continually distributed mass is replaced with a consistent mass (Vasiljević et al., 2013). The consistent mass matrix of the system is formulated on the basis of the same interpolation functions which are used for derivation of stiffness matrices. The threedimensional linear beam is modelled by means of a space beam element with two node points and six unknowns in each node. In this paper, the formulated models include damping of the portal structure. Including selected references on the crane modelling and dynamics (Gustafsson, 1996; Maczyński and Szczotka, 2002), which were considered in Vasiljević et al. (2016), in this paper additional references on the effect of structure damping (Chopra, 2006; Spyrakos, 1994; Bathe, 1996) and on the dynamic stability of cranes (Ito et al., 1985; Towarek, 1998; Ju et al., 2006; Shaikh and Kumar, 2016) are considered.

Results of dynamic analysis for two types of portal structures are presented and compared. Dynamic models of two portals of the $\mathrm{H}$ and $\mathrm{X}$ portal-slewing cranes are evaluated in terms modal analysis of vibration and the dynamic response of the portals to excitation.

\section{Kinematics of the portal cranes}

This paper assumes that the both portal cranes (H-type and X-type), i.e. the base of the cranes, can perform only rectilinear motion from point to point. A trapezoidal speed profile is most frequently used for calculation of moving cranes mechanisms, $v=$ linear, Fig. 1. This profile is identical for both $\mathrm{H}$ and $\mathrm{X}$ portal cranes. Besides the speed profile, there are three more profiles: triangular, stepped and notched. In this paper, it is adopted that during one cycle both portal cranes $(\mathrm{H}$ and $\mathrm{X})$ move along the track with the trapezoidal speed profile, because it represents a stricter criterion for analysis compared to other types of profiles.

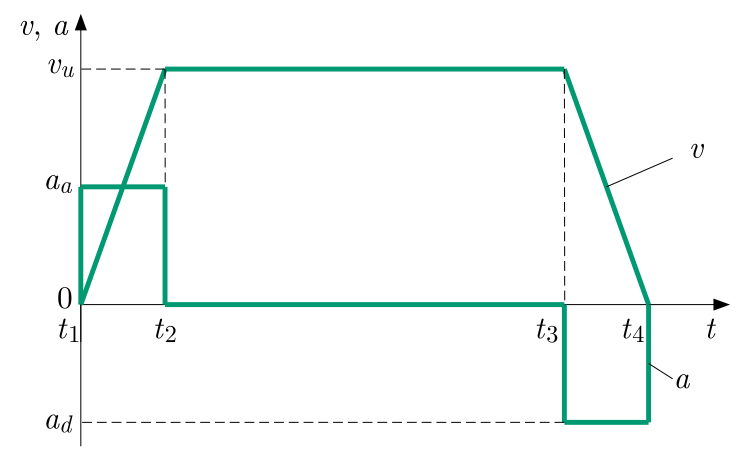

Fig. 1. Identical profiles of speed and acceleration for cranes type $\mathrm{H}$ and $\mathrm{X}$

\section{3D dynamic model of the general boom}

The identical general boom slewing is a constituent part of both types of portal-slewing cranes $(\mathrm{H}$ and $\mathrm{X})$. The subsystem of the boom consists of the portal of the boom and the rope system of the boom. The boom is connected to the platform by joints.

In the system of both portal cranes, the general boom slewing is observed as an independent subsystem which performs vibrations. An equivalent three-dimensional dynamic model of the general boom slewing of the portal crane (Fig. 2) was formed in Vasiljević et al. (2016). An 
equivalent dynamic model is formed in such a way that it keeps the main dynamic characteristics of the boom and that the defined problem could be mathematically solved.
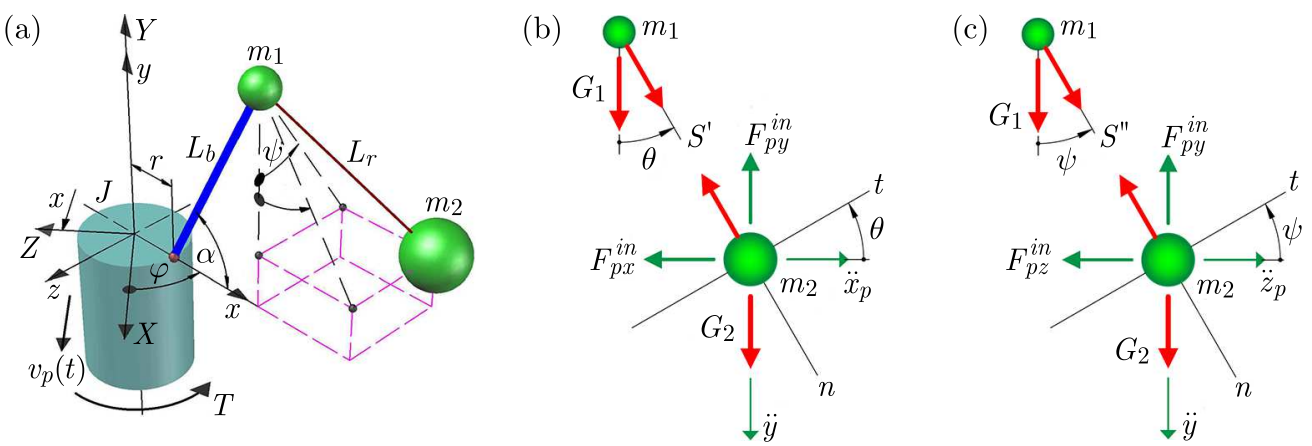

Fig. 2. (a) 3D dynamic model of the general boom slewing (identical for cranes of type $\mathrm{H}$ and $\mathrm{X}$ ),

(b) payload vibration in the longitudinal direction, (c) payload vibration in the lateral direction

This model was formed for the needs of determination of dynamic loads. From Fig. 2 it is seen that the subsystem of the boom with a payload is represented with two lumped masses, two lightweight bars and a circular disc. Discretization of the carrying structure of the boom with the mass $m_{b}$ was performed to a lightweight bar with the length $L_{b}$ and the reduced mass $m_{1}$ on the top. The reduced mass $m_{1}$ can be determined according to the following relation $m_{1}=(0.25$ $0.33) m_{b}$. The rope system of the boom is represented by a non-elastic lightweight bar with the length $L_{r}$ and the mass of the payload $m_{2}$. The slewing column is represented by a circular disc with the axial moment of inertia $J$ and the moment of rotation $T$. The distance between the boom joint and the column axis is $r$.

For the formed equivalent three-dimensional dynamic model boom (Fig. 2), the Lagrange equations of the second kind will be used for mathematical formulation (Vasiljević et al., 2016).

The dynamic differential equation of slewing of the column read

$$
J \ddot{\varphi}(t)=T
$$

The dynamic differential equations of vibrations of the payload reads

$$
\begin{aligned}
& L_{r} \ddot{\theta}(t)+g \sin \theta(t)=-\ddot{x}(t) \cos \theta(t) \cos \varphi \\
& L_{r} \ddot{\psi}(t)+g \sin \psi(t)=-\ddot{x}(t) \cos \psi(t) \sin \varphi
\end{aligned}
$$

where: $\theta$ - angle of payload vibration in the longitudinal direction ( $x y$-plane), $\psi$ - angle of payload vibration in the lateral direction ( $z y$-plane), $\varphi$ - angle of boom slewing, $x$ - rectilinear moving of the whole crane ( $X$-axis).

Differential equations (3.2) were solved analytically by using the Laplace transform method. These equations were verified by using the program written in Mathematica ${ }^{\circledR}$. An expanded setup process mathematical formulation was given in Vasiljević et al. (2016). The laws of payload vibration in the longitudinal and lateral direction are

$$
\begin{aligned}
\theta(t) & =-\frac{h}{L_{r} \omega^{2}}\left\{(1-\cos \omega t)-\left[1-\cos \omega\left(t-t_{2}\right)\right] \text { HeavisideTheta }\left(t-t_{2}\right)\right. \\
& -\left[1-\cos \omega\left(t-t_{3}\right)\right] \text { HeavisideTheta }\left(t-t_{3}\right) \\
& \left.+\left[1-\cos \omega\left(t-t_{4}\right)\right] \text { HeavisideTheta }\left(t-t_{4}\right)\right\} \cos \varphi \\
\psi(t) & =-\frac{h}{L_{r} \omega^{2}}\left\{(1-\cos \omega t)-\left[1-\cos \omega\left(t-t_{2}\right)\right] \text { HeavisideTheta }\left(t-t_{2}\right)\right. \\
& -\left[1-\cos \omega\left(t-t_{3}\right)\right] \text { HeavisideTheta }\left(t-t_{3}\right) \\
& \left.+\left[1-\cos \omega\left(t-t_{4}\right)\right] \text { HeavisideTheta }\left(t-t_{4}\right)\right\} \sin \varphi
\end{aligned}
$$


where: $h$-amplitude of input acceleration, $t_{i}$ - corresponding time impulses in the profiles of speed and acceleration (Fig. 1), $\omega$ - natural frequency of payload vibration $\left(\omega^{2}=g / L_{r}\right)$.

For Eqs. (3.3), the corresponding comments are provided in Vasiljević et al. (2016).

\section{Comparative 3D dynamic models of the $\mathrm{H}$ and $\mathrm{X}$ portals}

\subsection{Comparative description of the $\mathrm{H}$ and $\mathrm{X}$ portals}

Both types of portal cranes ( $\mathrm{H}$ and $\mathrm{X}$ ) are divided into two subsystems (moving structure and slewing boom). Taking into account that the boom rotates around its axis, it can be concluded that the planar model is not sufficient for describing the dynamic behaviour of the considered portal-slewing cranes. The three-dimensional dynamic models of the two portals, to which dynamic load is reduced, is formed in accordance with the 3D dynamic model of the boom.

The comparative discretized 3D models of the portals are shown in Fig. 3. The both types of portals $(\mathrm{H}, \mathrm{X})$ are rigid three-dimensional frames. The base of the portals has identical dimensions $L \times B$. The main structural parts of both portals are legs, slanted columns, frame ( $\mathrm{H}$ or $\mathrm{X}$ ) and circular ring. The legs of both portals are identical and stand at the same level. The slanted columns are identical and they are connected to the legs as well as to the frame ( $\mathrm{H}$ or $\mathrm{X})$ and the circular ring. The distance between the frames and the circular ring is $H_{s}$.

(a)

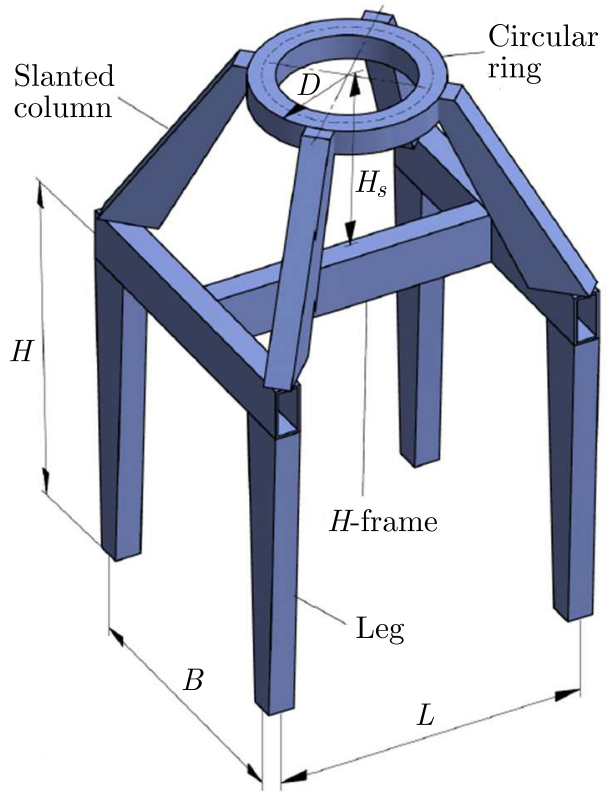

(b)

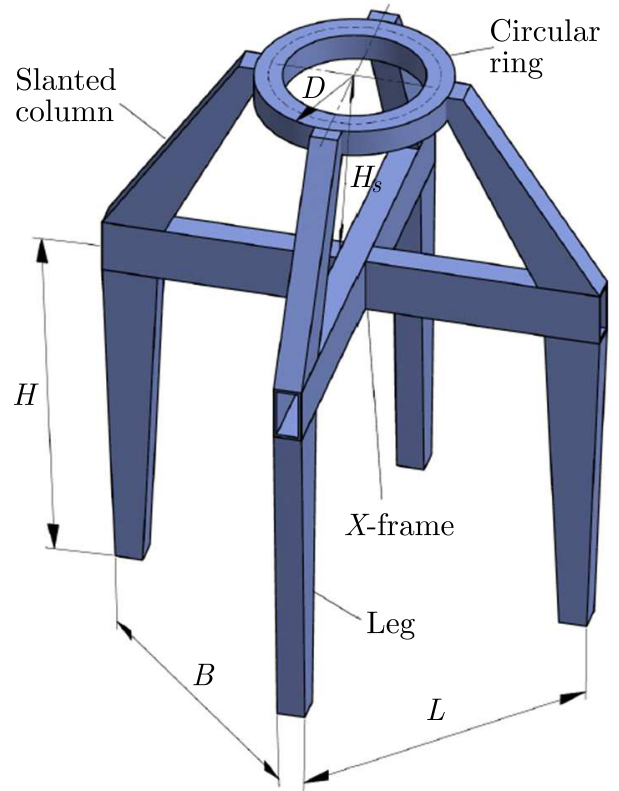

Fig. 3. Comparison of discretized 3D models of the portals: (a) type H, (b) type X

\subsection{Comparative 3D FE models of the portals of $H$ and $X$ types}

The interaction between the structure and the boom is simplified in such a way that the influence of the payload and the dead weight of the boom are reduced to the points of the upper and lower supports of the boom. A three-dimensional dynamic model of the $\mathrm{H}$ portal of the portal-slewing crane for forced undamped vibrations was formed in Vasiljević et al. (2016). In this paper, an advanced dynamic model of the $\mathrm{H}$ portal for forced damped vibrations including damping of the portal structure is formed (Fig. 4a). Analogously, a 3D dynamic model of the $\mathrm{X}$ portal has been given (Fig. 4b). 

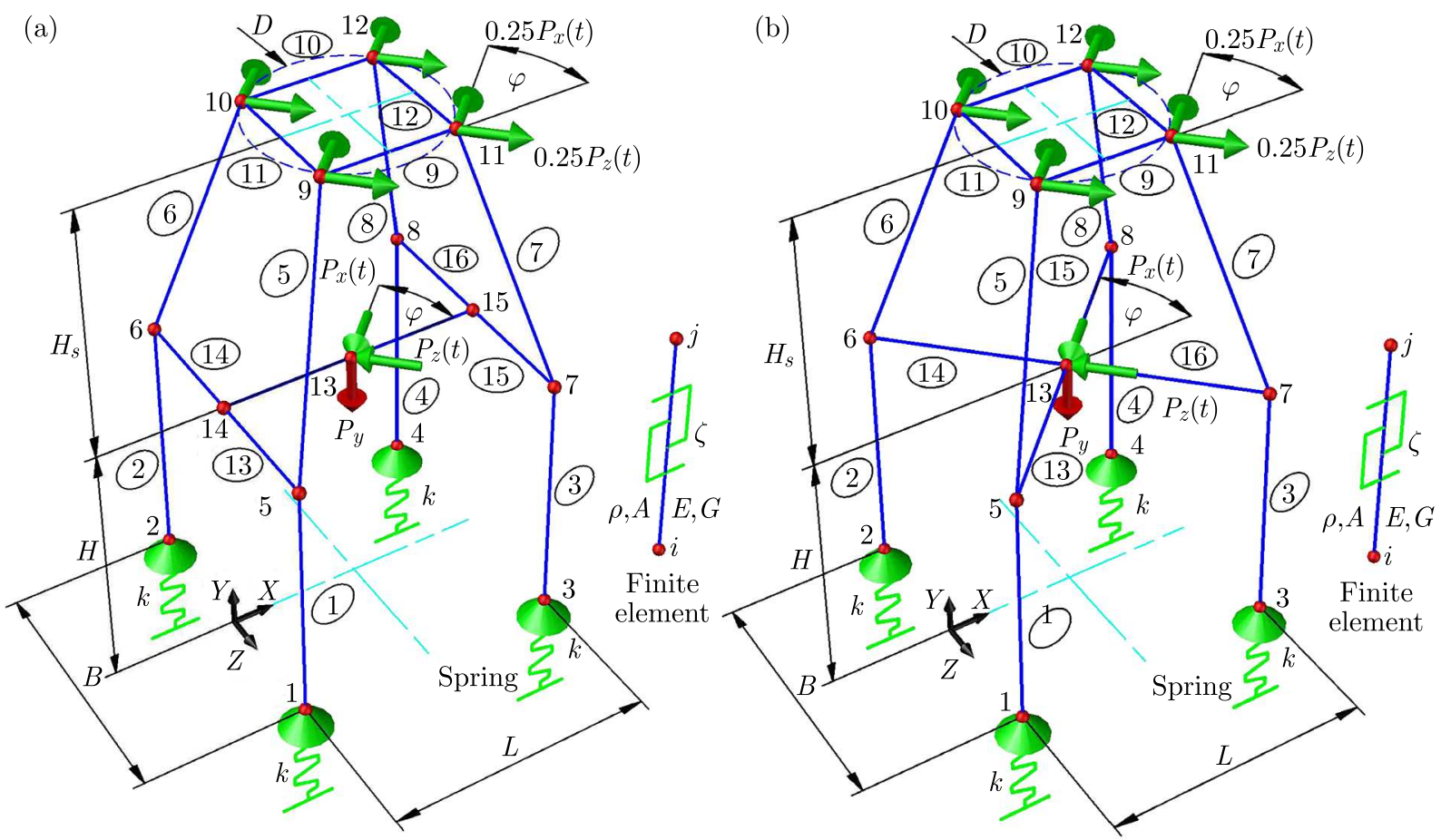

Fig. 4. 3D FE models of the portals: (a) type H, (b) type $\mathrm{X}$

The portal structure of the type $\mathrm{H}$ was discretized into 18 finite elements of the beam type with 6 degrees of freedom in the node. In other words, the portal was discretized into 18 finite beam elements connected by nodes. The portal $\mathrm{H}$ (whole system) has 82 degrees of freedom. The frame $\mathrm{H}$ was divided into 6 finite elements. The circular ring with diameter $D$ was divided into 4 finite elements. The legs and the slanted columns were modelled as 1 finite element.

Analogically, the portal structure of the type $\mathrm{X}$ was discretized into 16 finite beam with 6 degrees of freedom in the node, i.e., the portal was discretized into 16 finite beam elements connected by nodes. The portal $\mathrm{X}$ has 70 degrees of freedom. The frame $\mathrm{X}$ was divided into 4 finite elements. In the portal X, the circular ring, legs and slanted columns were divided into the same number of finite elements, as in the portal $\mathrm{H}$.

In both portals the finite elements have properties $A_{n}, I_{x n}, I_{y n}, I_{z n}(n=1, \ldots, 18$ - type $\mathrm{H}$; $n=1, \ldots, 16$ - type $\mathrm{X}$ ). The both FE models are rest on four supports (springs) with the stiffness $k$. The main mechanical properties of all finite elements of the three-dimensional model of the steel-made portal are the elastic modulus $E$, shear modulus $G$, mass density $\rho$ and the damping coefficient $\xi$. The models include damping within the portal structure in the range of 0.5-7\% (Chopra, 2006). In the dynamic analysis with the application of the FEM, the maximum magnitude of damping is 7\% (Spyrakos, 1994).

The dynamic moments are reduced to couples of horizontal dynamic forces $P_{x}(t)$ and $P_{z}(t)$ in the nodes of finite elements of the upper beam and the central node of the lower beam (case 1: frame - type $\mathrm{H}$, case 2: frame - type X). The position of these forces relative to the longitudinal direction (X-axis) is defined by the angle $\varphi$ which represents the angle of platform slewing.

In the longitudinal and lateral direction, horizontal dynamic force components $\left(P_{x}(t)\right.$, $4 \cdot 0.25 P_{x}(t)$ and $\left.P_{z}(t), 4 \cdot 0.25 P_{z}(t)\right)$ make couples of forces which oppose dynamic moments components of overturning $\left(M_{z}(t)\right.$ and $\left.M_{x}(t)\right)$ of the platform 


$$
\begin{aligned}
& P_{x}(t)=\frac{M_{z}(t)}{H_{s}}=\frac{1}{H_{s}}\left[m_{1} g\left(L_{b} \cos \alpha+r\right)+m_{2}\left(g+L_{r} \dot{\theta}^{2} \cos \theta\right)\left(L_{r} \sin \theta+L_{b} \cos \alpha+r\right)\right. \\
& \left.\quad+m_{2} L_{r} \dot{\theta}^{2} \sin \theta\left(L_{b} \sin \alpha-L_{r} \cos \theta\right)+m_{2} L_{r} \dot{\psi}^{2} \cos \psi\left(L_{b} \cos \alpha+r\right)\right] \\
& P_{z}(t)=\frac{M_{x}(t)}{H_{s}}=\frac{1}{H_{s}}\left[m_{2}\left(g+L_{r} \dot{\psi}^{2} \cos \psi\right) L_{r} \sin \psi+m_{2} L_{r} \dot{\psi}^{2} \sin \psi\left(L_{b} \sin \alpha-L_{r} \cos \psi\right)\right]
\end{aligned}
$$

The vertical static force $P_{y 1}$ of the reduced boom mass $m_{1}$ and the vertical static force $P_{y 2}$ of the load mass $m_{2}$ are reduced to the central node of the lower beam (frame - type $\mathrm{H}$ and frame - type X). The total vertical static force $P_{y}$ which acts in the central node of the lower support of the boom is equal to the sum of the two forces $\left(P_{y 1}\right.$ and $\left.P_{y 2}\right)$

$$
P_{Y}=P_{y}=P_{y_{1}}+P_{y_{2}}
$$

For testing dynamic stability of both portals to identical excitation, characteristic nodes of the portal models are the nodes at the point of supports (springs).

\subsection{Comparative of mathematical formulation}

In accordance with the dynamical models of the two portals, the differential equation of dynamic equilibrium, i.e. forced damped vibrations of the portals reads

$$
\mathbf{M} \ddot{\mathbf{U}}+\mathbf{C} \dot{\mathbf{U}}+\mathbf{K U}=\mathbf{P}(t)
$$

where: $\mathbf{M}$ - matrix of consistent masses of the system, $\mathbf{C}$ - matrix of damping, $\mathbf{K}$ - matrix of stiffness, $\ddot{\mathbf{U}}$ - acceleration vector, $\dot{\mathbf{U}}$ - speed vector, $\mathbf{U}$ - displacement vector, and $\mathbf{P}(t)-$ external force vector.

Natural frequencies of the system are obtained by solving the algebraic equation

$$
\operatorname{det}\left(\mathbf{K}-\omega_{p}^{2} \mathbf{M}\right)=0
$$

The mass matrix of the whole systems, i.e. the portals of type $\mathrm{H}$ and $\mathrm{X}$ reads

$$
\mathbf{M}= \begin{cases}{[\mathbf{M}]_{82 \times 82}} & \text { type } \mathrm{H} \\ {[\mathbf{M}]_{70 \times 70}} & \text { type } \mathrm{X}\end{cases}
$$

The stiffness matrix of the portals and springs is

$$
\mathbf{K}= \begin{cases}{[\mathbf{K}]_{82 \times 82}+\mathbf{k}_{16 \times 16}} & \text { type } \mathrm{H} \\ {[\mathbf{K}]_{70 \times 70}+\mathbf{k}_{16 \times 16}} & \text { type } \mathrm{X}\end{cases}
$$

In order to execute Eqs. (4.6), the stiffness matrix $\left[\mathbf{k}_{s}\right]_{16 \times 16}$ is, by adding zero rows and zero columns ( $\mathrm{Wu}, 2004)$, extended to the matrix with dimensions $82 \times 82-$ for type $\mathrm{H}$ and $70 \times 70$ - for type X, except for 16 degrees of freedom of 4 nodes.

Structural damping of the portal is modelled by conversion to the equivalent viscous damping. The damping matrix has been adopted according to the Rayleigh theory (Chopra, 2006)

$$
\mathbf{C}_{p}=\alpha \mathbf{M}_{p}+\beta \mathbf{K}_{p}
$$

where $\alpha$ and $\beta$ are damping factors. Details of determining the damping factors are given in Chopra (2006), Bathe (1996). In this paper, the assumption is that the damping coefficients for the control frequencies, $\omega_{p 1}$ and $\omega_{p 2}$, are the same $\left(\xi=\xi_{1}=\xi_{2}\right)$. The simplified form of the equation for determining the damping factor is

$$
\left\{\begin{array}{l}
\alpha \\
\beta
\end{array}\right\}=\frac{2 \xi}{\omega_{p 1}+\omega_{p 2}}\left\{\begin{array}{c}
\omega_{p 1} \omega_{p 2} \\
1
\end{array}\right\}
$$


The damping matrix of the portal structure, i.e. the overall system is

$$
\mathbf{C}= \begin{cases}{\left[\mathbf{C}_{p}\right]_{82 \times 82}} & \text { type } \mathrm{H} \\ {\left[\mathbf{C}_{p}\right]_{70 \times 70}} & \text { type } \mathrm{X}\end{cases}
$$

The external force vector of the portal structure, i.e. the overall system and the springs is

$$
\mathbf{P}(t)= \begin{cases}\left\{\mathbf{P}_{p}(t)\right\}_{82 \times 1} & \text { type } \mathrm{H} \\ \left\{\mathbf{P}_{p}(t)\right\}_{70 \times 1} & \text { type } \mathrm{X}\end{cases}
$$

The vectors of external dynamic forces $\mathbf{P}(t)$ can be expressed in the following forms:

- type $\mathrm{H}$

$$
\mathbf{P}(t)^{\mathrm{T}}= \begin{cases}\{0,0,0,0,0,0\} & i=1, \ldots, 4 \\ \{0,0,0,0,0,0\} & i=5, \ldots, 8 \\ \left\{0.25 P_{X}(t), 0.25 P_{Z}(t), 0,0,0,0\right\} & i=9, \ldots, 12 \\ \left\{-P_{X}(t),-P_{Z}(t), P_{Y}, 0,0,0\right\} & i=13 \\ \{0,0,0,0,0,0\} & i=14,15\end{cases}
$$

- type X

$$
\mathbf{P}(t)^{\mathrm{T}}= \begin{cases}\{0,0,0,0,0,0\} & i=1, \ldots, 4 \\ \{0,0,0,0,0,0\} & i=5, \ldots, 8 \\ \left\{0.25 P_{X}(t), 0.25 P_{Z}(t), 0,0,0,0\right\} & i=9, \ldots, 12 \\ \left\{-P_{X}(t),-P_{Z}(t), P_{Y}, 0,0,0\right\} & i=13\end{cases}
$$

where

$$
P_{X}(t)=P_{x}(t) \cos \varphi+P_{z}(t) \sin \varphi \quad P_{Z}(t)=-P_{x}(t) \sin \varphi+P_{z}(t) \cos \varphi
$$

\subsection{Solving the equation of dynamic equilibrium}

As the equation of dynamic equilibrium (Eq. (4.3)) represents a differential equation of the second order with variable coefficients, its solving is complex. That is the reason why a short procedure of solving the equation of dynamic equilibrium is given here in 6 steps.

Step (i) - Determination of the matrices of stiffness $\mathbf{K}$ and inertia $\mathbf{M}$ of the two finite element models of the portals (type $\mathrm{H}$ and type $\mathrm{X}$ ) of the considered cranes.

Step (ii) - Determination of natural frequencies of the two FE models by using Eq. (4.4) and then the first two lowest natural frequencies $\omega_{p 1}$ and $\omega_{p 2}$.

Step (iii) - Determination of the matrix of damping $\mathbf{C}$ of the two FE models.

Step (iv) - Determination of the external vectors of forces $\mathbf{P}(t)$ as a function of time $t$.

Step (v) - Division of the shared total time domain $T$ into $n$ steps in such a way to obtain the corresponding time interval $\Delta t$.

Step (vi) - Solving Eq. (4.3) for each time step $n$, where $n$ takes magnitudes from 0 to $p$. A special case of the Newmark method, "the method of average acceleration" is used.

\section{Numerical example of comparative dynamic analysis}

In this Section, a solution for portal cranes to apply the theoretical dynamic analysis presented in Sections 2, 3 and 4 for checking its correctness and drawing the corresponding conclusions is used. Numerous examples are carried out for the portal cranes of the type $\mathrm{H}$ and $\mathrm{X}$ with the input data given in Tables 1 and 2. 
Table 1. Identical general data for portal cranes of the type $\mathrm{H}$ and $\mathrm{X}$

\begin{tabular}{|l|c|}
\hline \multicolumn{1}{|c|}{ Crane properties } & Value \\
\hline \hline Payload $m_{Q}[\mathrm{t}]$ & 10 \\
\hline Speed of crane $v_{p}[\mathrm{~m} / \mathrm{min}]$ & 30 \\
\hline Length of portal base $L[\mathrm{~m}]$ & 6 \\
\hline Width of portal base $B[\mathrm{~m}]$ & 6 \\
\hline Height of leg $H[\mathrm{~m}]$ & 6 \\
\hline Distance between supports and boom $H_{s}[\mathrm{~m}]$ & 3.675 \\
\hline Diameter of circular ring $D[\mathrm{~m}]$ & 4.243 \\
\hline Boom mass $m_{b}[\mathrm{~kg}]$ & 9200 \\
\hline Boom length $L_{b}[\mathrm{~m}]$ & 30 \\
\hline Distance between boom joint and column $r[\mathrm{~m}]$ & 1 \\
\hline Modulus of elasticity $E\left[\mathrm{~N} / \mathrm{mm}^{2}\right]$ & $2.1 \cdot 10^{11}$ \\
\hline Modulus of shear $G\left[\mathrm{~N} / \mathrm{mm}{ }^{2}\right]$ & $0.8 \cdot 10^{11}$ \\
\hline Mass density of material $\rho\left[\mathrm{kg} / \mathrm{m}^{3}\right]$ & 7850 \\
\hline Stiffness of elastic supports $k[\mathrm{~N} / \mathrm{m}]$ & $1 \cdot 10^{7}$ \\
\hline Coefficient of relative damping $\xi[\%]$ & 0.5 \\
\hline
\end{tabular}

Table 2. Comparative of dimensions of the cross-sections of the $\mathrm{H}$ and $\mathrm{X}$ portals

\begin{tabular}{|c|c|c|c|c|c|c|}
\hline \multirow{2}{*}{ Cross-section } & Type $\mathrm{H}$ & $n$ & $1, \ldots, 4$ & $5, \ldots, 8$ & $9, \ldots, 12$ & $13, \ldots, 16$ \\
\cline { 2 - 7 } & Type X & $n$ & $1, \ldots, 4$ & $5, \ldots, 8$ & $9, \ldots, 12$ & $13, \ldots, 18$ \\
\hline \hline \multirow{2}{*}{$H_{n}[\mathrm{~mm}]$} & $i$ & 530 & 1030 & 524 & 530 \\
\cline { 2 - 7 } & $j$ & 1030 & 530 & 524 & 530 \\
\cline { 2 - 7 } & \multirow{2}{*}{$H_{n 1}[\mathrm{~mm}]$} & $i$ & 500 & 1000 & 500 & 500 \\
\cline { 3 - 7 } & $j$ & 1000 & 500 & 500 & 500 \\
\cline { 2 - 7 } & \multirow{2}{*}{$B_{n}[\mathrm{~mm}]$} & $i$ & 550 & 550 & 550 & 550 \\
\cline { 3 - 7 } & & $j$ & 550 & 550 & 550 & 550 \\
\cline { 2 - 7 } & \multirow{2}{*}{$B_{n 1}[\mathrm{~mm}]$} & $i$ & 500 & 500 & 500 & 500 \\
\cline { 2 - 7 } & \multirow{2}{*}{$\delta_{1}[\mathrm{~mm}]$} & $i$ & 500 & 500 & 500 & 500 \\
\cline { 2 - 7 } & & $j$ & 10 & 10 & 12 & 10 \\
\cline { 2 - 7 } & \multirow{2}{*}{$\delta_{2}[\mathrm{~mm}]$} & $i$ & 15 & 15 & 12 & 12 \\
\cline { 3 - 7 } & $j$ & 15 & 15 & 12 & 15 \\
\hline
\end{tabular}

\subsection{Excitation}

The motion of both types of cranes along the tracks is described by the diagram of speed (Fig. 5). The speed $v_{p 1}$ is the real nominal speed, whereas the speed $v_{p 2}$ is the extreme nominal speed of the portal. The impact of the magnitudes of speed on the dynamic response of the payload was determined. Figure 6 based on Eq. (3.3) $)_{1}$ presents the angle of payload vibration $\theta$ in the longitudinal direction for two speeds of both portals. This vibration diagram shows that the angle of payload vibration increases with the increase in the speed from real to extreme values. The maximum angle of payload vibration for the speed $v_{p 1}$ is $0.0693 \mathrm{rad}$. The change of the angle of payload vibration $\psi$ in the lateral direction is completely the same. The change of the dynamic force component in the longitudinal direction, on the basis of Eq. $(4.1)_{1}$, is presented in Fig. 7. The change of the dynamic force component in the lateral direction, on the basis of Eq. (4.1) $)_{2}$, is presented in Fig. 8. Figures 5-8 are identical for both portal cranes. 


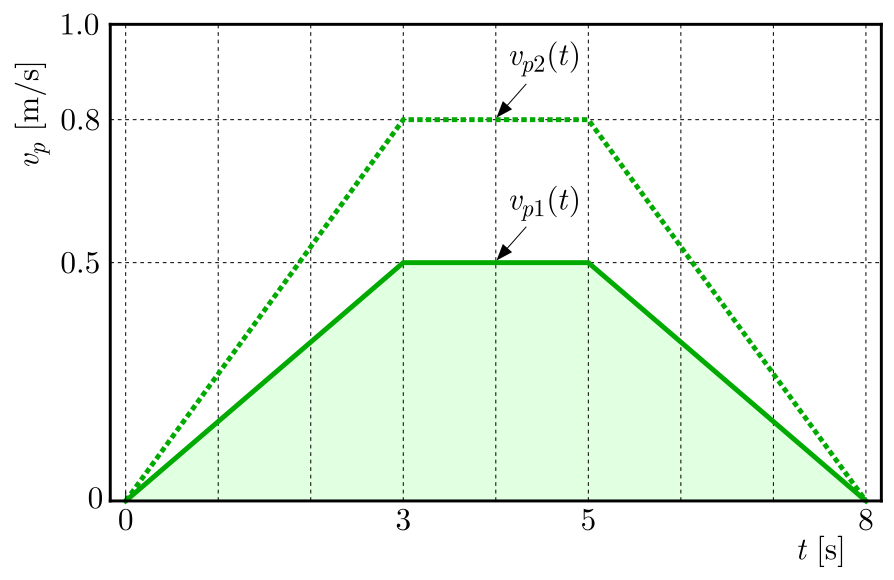

Fig. 5. Time diagram of the portal speed $v_{p}(t)$

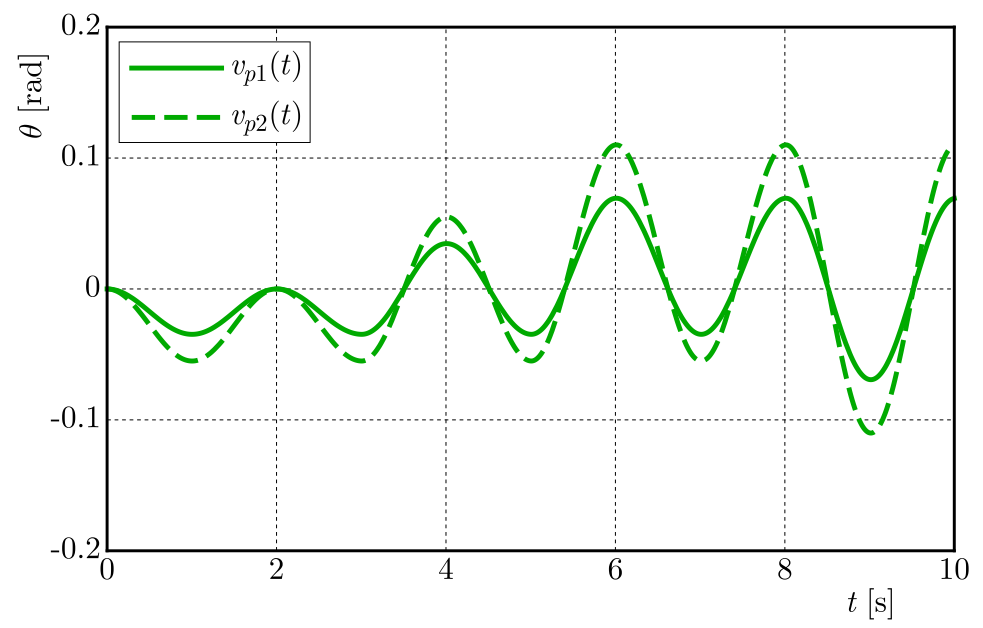

Fig. 6. Angle of payload vibration in the longitudinal direction for different speeds of crane motion

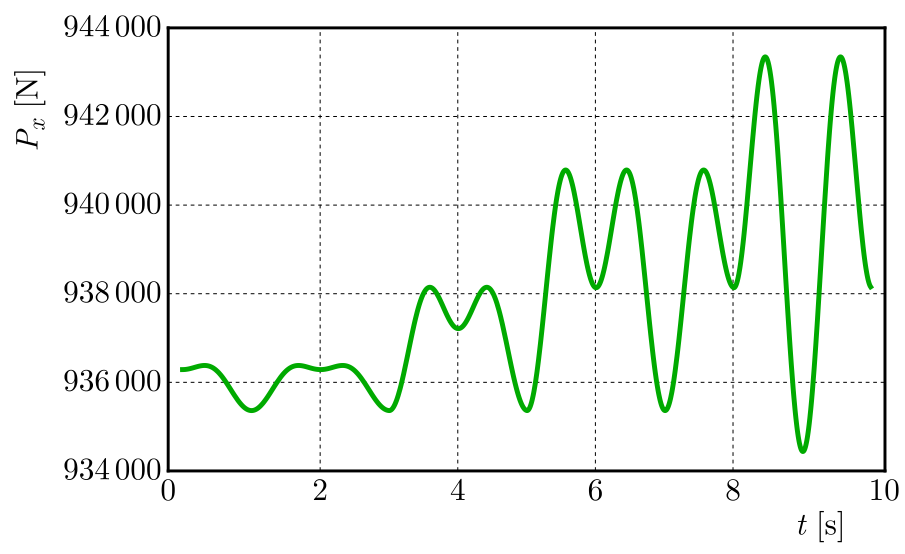

Fig. 7. Change of the dynamic force component in the longitudinal direction

\subsection{Comparative modal analysis}

The first main intention of this paper is comparison of modal parameters (eigenfrequencies and vibration modes) between the two types of portal cranes (type $\mathrm{H}$ and $\mathrm{X}$ ). Although both portals have the same spread and height, there are differences in modal parameters. These differences come from the difference in their frames at the medium level in the horizontal plane. 


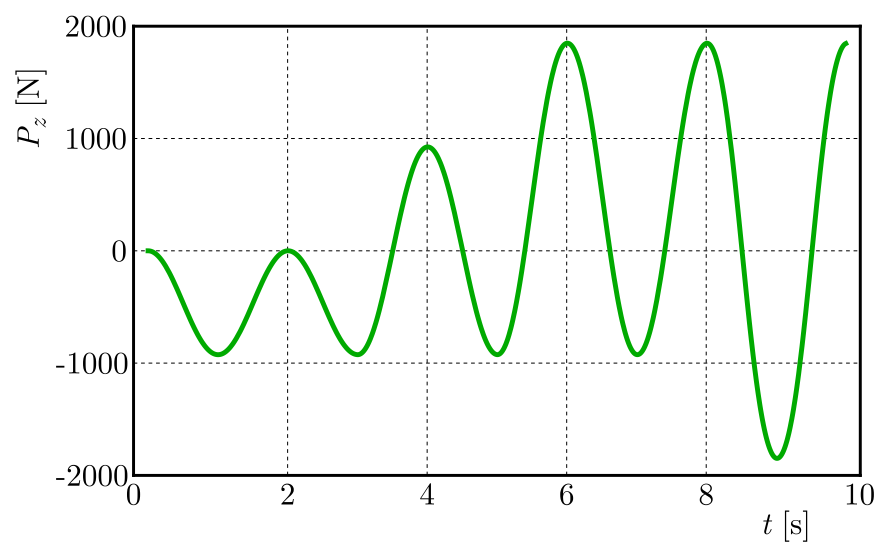

Fig. 8. Change of the dynamic force component in the lateral direction

Table 3 presents a comparison of the magnitudes of the first six comparative frequencies and periods of vibration of the two types of portals of the portal-slewing crane (Eq. (4.4)). Also, the relative differences of these parameters are given.

Table 3. Comparison of modal parameters of vibration of the two portals

\begin{tabular}{|c|l|c|c|c|c|c|c|}
\hline Portal & \multicolumn{1}{|c|}{ Mode } & 1 & 2 & 3 & 4 & 5 & 6 \\
\hline \hline Type H & Natural frequency $f_{H}[\mathrm{~Hz}]$ & 2.72 & 2.92 & 5.26 & 7.91 & 13.66 & 16.04 \\
\hline Type X & Natural frequency $f_{X}[\mathrm{~Hz}]$ & 2.87 & 2.89 & 4.92 & 7.92 & 15.31 & 15.37 \\
\hline & Relative difference $[\%]$ & -7.66 & 3.34 & 16.04 & -0.88 & -7.03 & 12.52 \\
\hline
\end{tabular}

Based on Table 3, it is concluded that the first 6 frequencies are slightly different. For the construction, the most dangerous are the first two or three major forms of vibration, since there are the greatest amplitudes quickly leading to failure of the structure (Petković, 1996; Ju et al., 2006). In this regard, when comparing portals of the type $H$ and $X$, the accent is on the first two modes of vibration.

As an imperative to carrying transport structures, building and mining machinery, small mass, and slenderness it is common that the first lowest (basic) frequency is in the range of [0.5-3.5] Hz (Petković, 1996). In this aspect, for both types of portals, the first frequency is in the recommended range. The portal $\mathrm{H}$ has a $7.66 \%$ lower first vibration frequency than the portal $\mathrm{X}$, which means that the portal $\mathrm{H}$ is more elastic from the aspect of the first vibration mode. The first vibration frequency of both portals is located in the defined range.

The portal $\mathrm{H}$ has a $3.44 \%$ higher second vibration frequency than the portal $\mathrm{X}$. The first and second mode shapes of vibration for both portals are shown Figs. 9 and 10. It can be seen that the first mode of vibration is dominated by deformation of both considered types of portals. This vibration mode is characterized by a horizontal shape of vibration. Also, the second vibration mode has the same character.

Dynamic behaviour of the construction is evaluated by the first vibration mode. Stable dynamic behaviour of a construction requires a high first vibration frequency. According to this aspect, the portal $\mathrm{H}$ is characterized by unfavourble dynamic stability compared to the portal $\mathrm{X}$.

\subsection{Comparative dynamic displacements}

The second main intention of the paper is to compare dynamic displacements of the two types of portals. Equation of dynamic equilibrium (Eq. (4.3)) for investigating the dynamics of the two portal cranes was solved numerically by using a direct integration method (step by step) and by using a program written in Mathematica ${ }^{\circledR}$. This program was written based on 

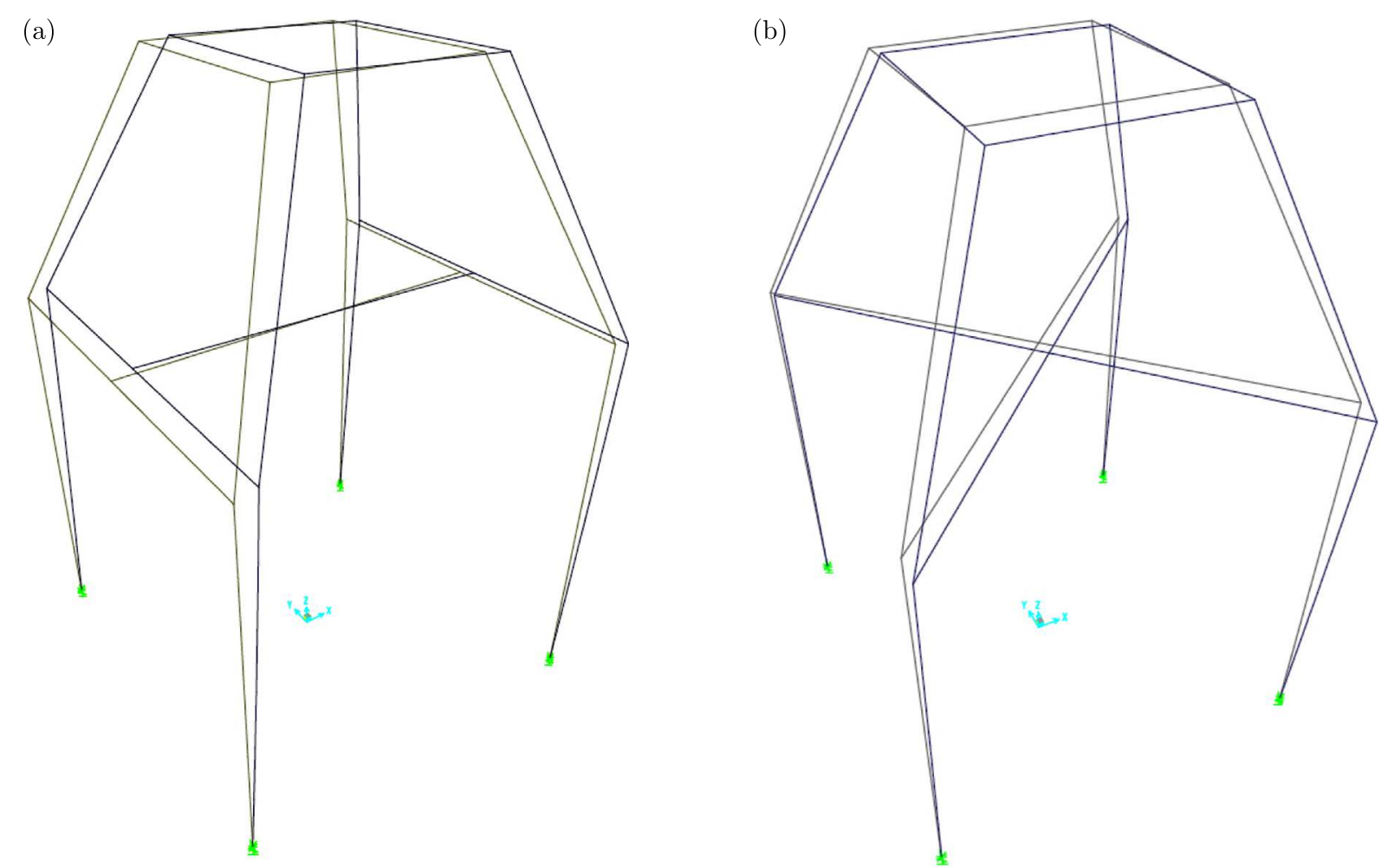

Fig. 9. Comparison of the 1st mode shape for two portals: (a) type H, (b) type X
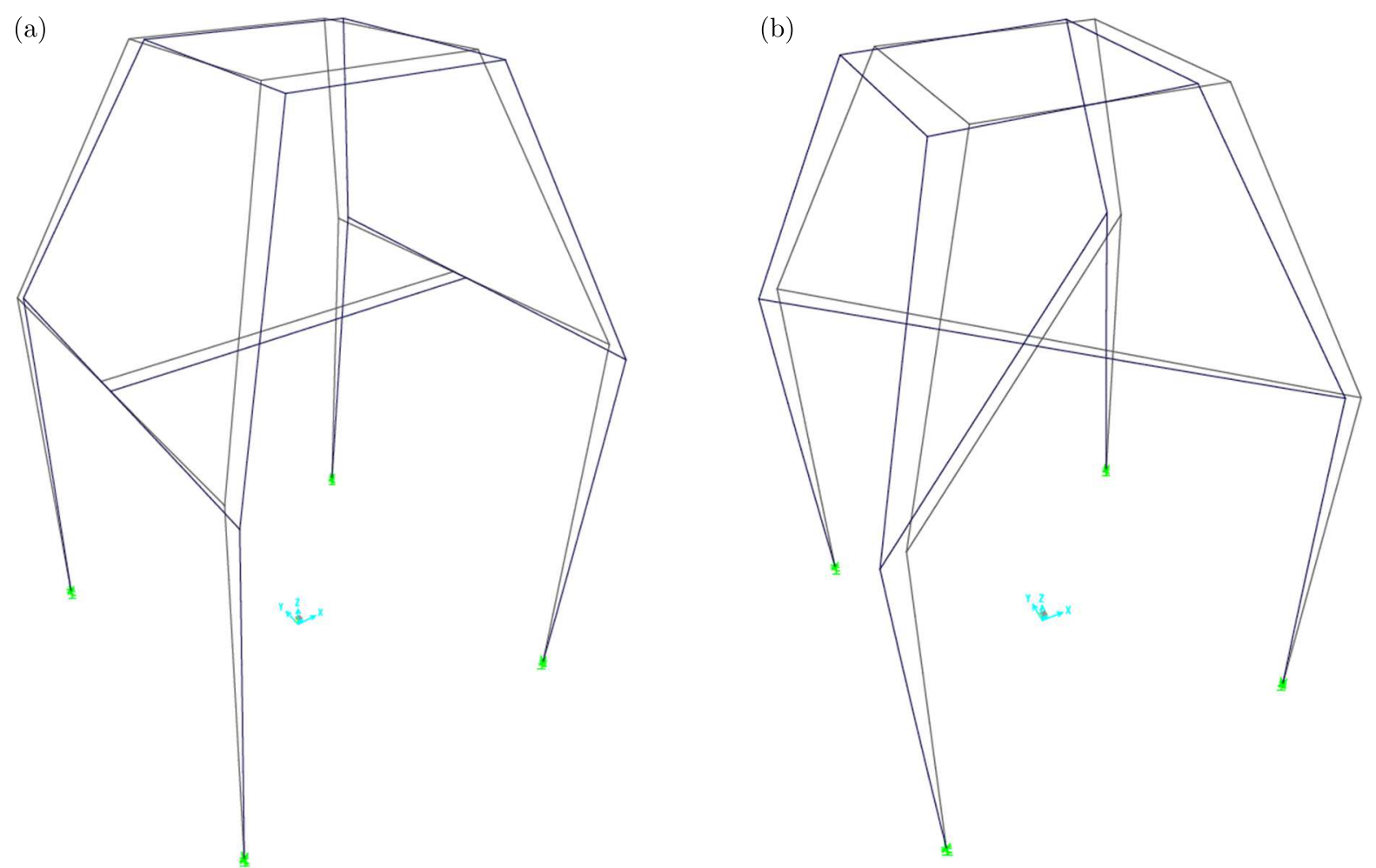

Fig. 10. Comparison of the 2nd mode shape for two portals: (a) type H, (b) type X 
the Newmark integration method (Bathe, 1996). The time interval of integration was chosen to be $\Delta t=0.01 \mathrm{~s}$. Dynamic displacements for all degrees of freedom were obtained, but only the most important ones for testing the dynamic stability of the two portal cranes are presented.

The dynamic responses of the portals $\mathrm{H}$ and $\mathrm{X}$ to identical excitation are, above all, contained in the dynamic displacement of the four supports (springs) for different positions of the boom relative to the direction of motion of the crane. Comparative analysis of dynamic displacements of the four springs i.e. nodes (1 through 4 ) for different positions of the boom relative to rectilinear motion of the portal $\varphi=\left(0^{\circ}, 45^{\circ}, 90^{\circ}, 135^{\circ}, 180^{\circ}\right)$ established that the critical position of the boom is $\varphi=45^{\circ}$ for both portals (type $\mathrm{H}$ and type $\mathrm{X}$ ). For this position of the boom, spring node 1 for both portals ( $\mathrm{H}$ and $\mathrm{X}$ ) has a positive displacement, which is bigger than positive displacements of springs for all other positions of the boom. In other words, the largest vertical displacement has the leg located on the opposite part of the portal structure in both axes in relation to the boom slewing of $45^{\circ}$ in relation to the rectilinear motion of the crane.

A comparative change of dynamic displacements of the four springs i.e. nodes ( 1 through 4$)$ for the critical position of the boom relative to the rectilinear motion of the portal $\mathrm{H}\left(\varphi=45^{\circ}\right)$ is shown in Fig. 11. Analogically, a corresponding analysis for the portal X is shown in Fig. 12.

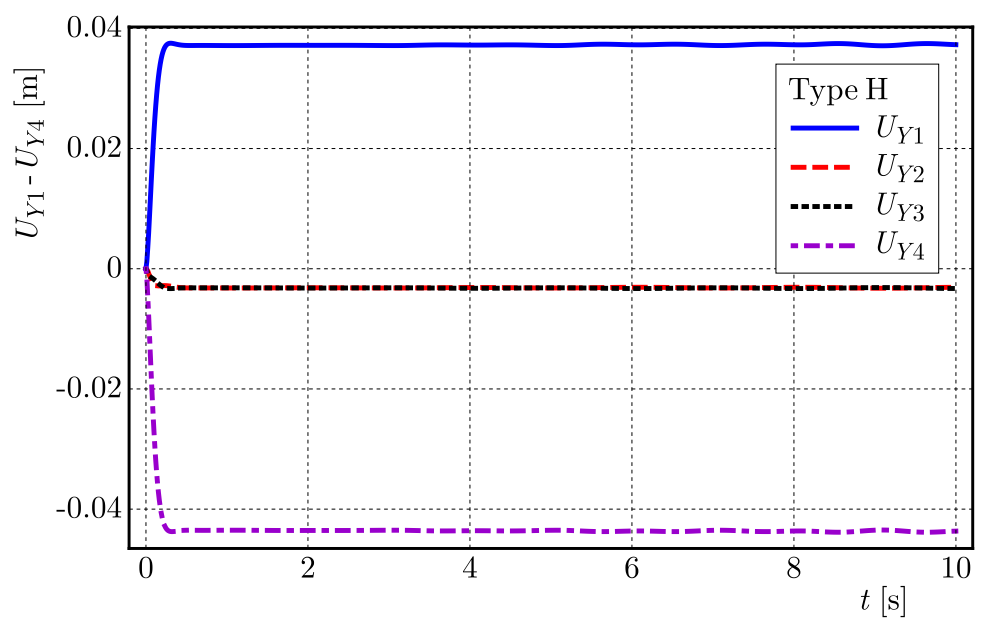

Fig. 11. Comparison of dynamic displacements of nodes 1 through 4 of the portal $\mathrm{H}$

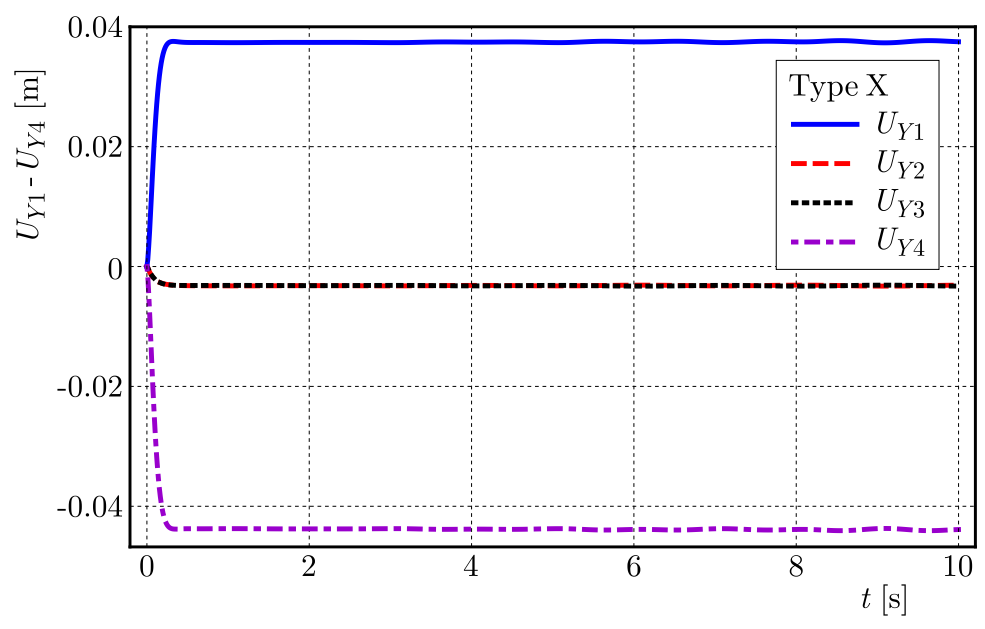

Fig. 12. Comparison of dynamic displacements of nodes 1 through 4 of the portal X 
Individual comparative changes of the dynamic displacements of node 1 for the defined critical position of the boom $\left(\varphi=45^{\circ}\right)$ for two portals $\mathrm{H}$ and $\mathrm{X}$ are shown in Figs. 13 through 16.

Based on the analysis of the displacements of the elastic supports for the critical position of the boom, Figs. 13 through 16, it is shown that only node 1 has a positive displacement (Fig. 13). For the portal H, the maximum displacement of node 1 is $37.52 \mathrm{~mm}$. For the portal X, the maximum displacement of node 1 is $37.69 \mathrm{~mm}$. The portal $\mathrm{H}$ has a $0.45 \%$ less dynamic displacement than the portal $\mathrm{X}$, which means that the portal $\mathrm{X}$ is has a unfavorable dynamic stability for overturn compared to the portal H. In this case, lifting of the leg of both portalslewing cranes occurs in practice most often. In accordance with the parameters included in the mathematical model of the considered portal, the expected magnitude of the maximum dynamic displacement was obtained.

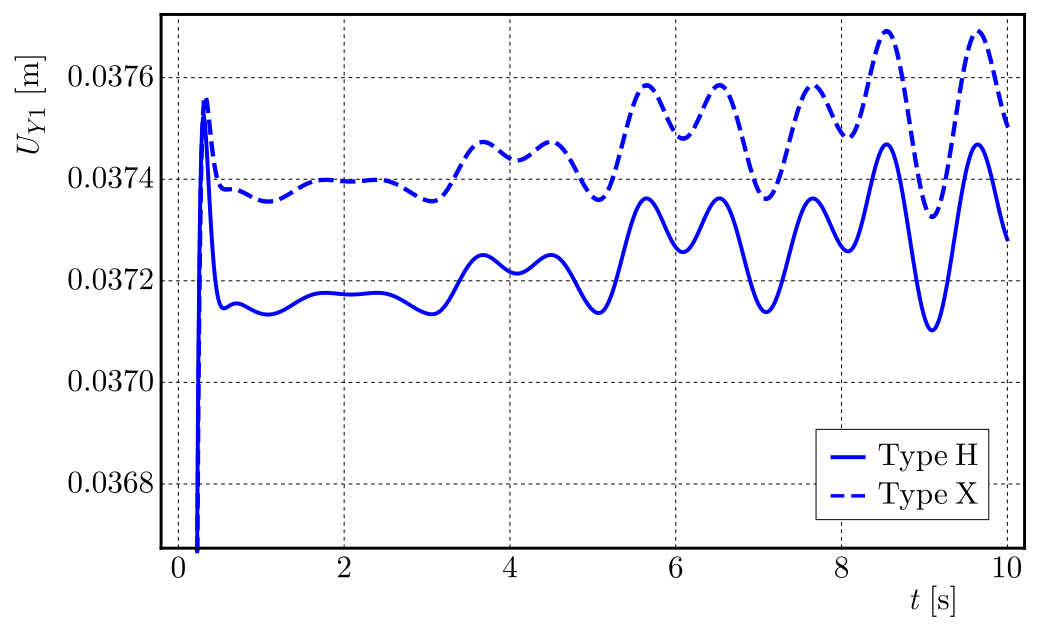

Fig. 13. Comparison of the dynamic displacement of node 1 for the two portals

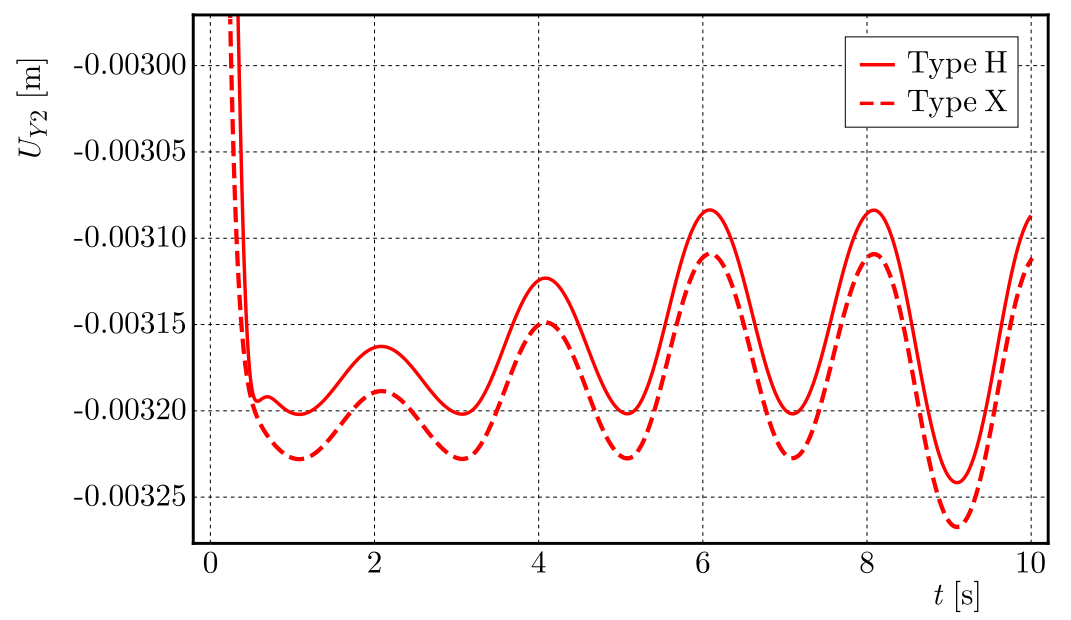

Fig. 14. Comparison of the dynamic displacement of node 2 for the two portals

Namely, in support 1 of the portal structure there is a clearance in terms of the track. Due to the clearance $U_{Y 1}$, the real value of pressure in support 1 is defined by the following expression $R_{1}=R_{s 1}-k U_{Y 1}$, where: $R_{s 1}$ is the pressure in support 1 when all supports lie in the tracks and $k$ is the equivalent rigidity of the portal structure and supporting ground. It is obvious that the pressure force is decreased in support 1 of the portal structure. 


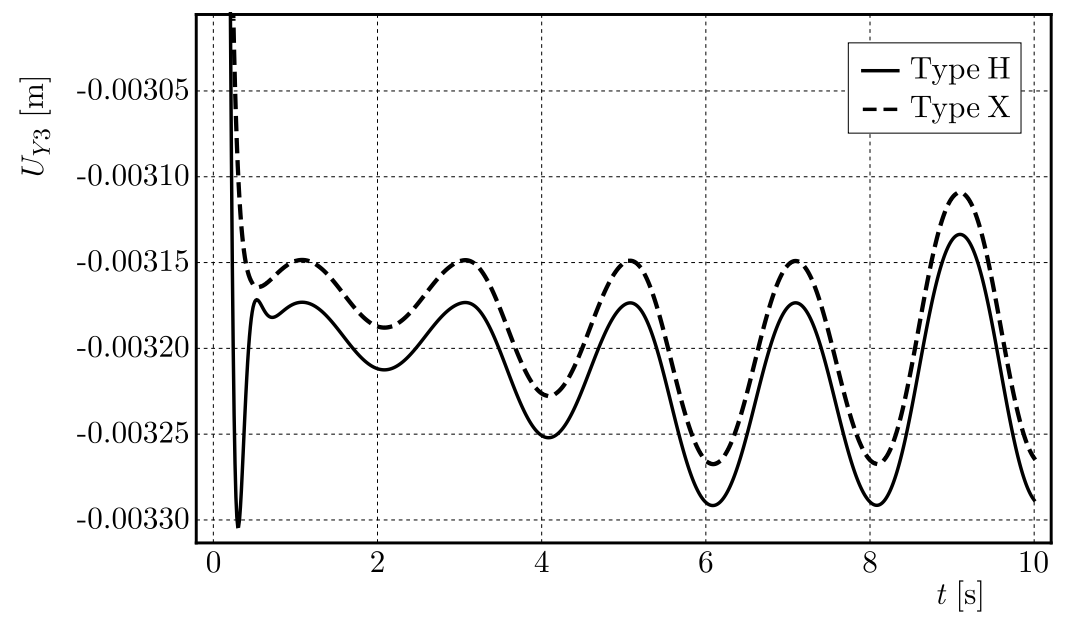

Fig. 15. Comparison of the dynamic displacement of node 3 for the two portals portals

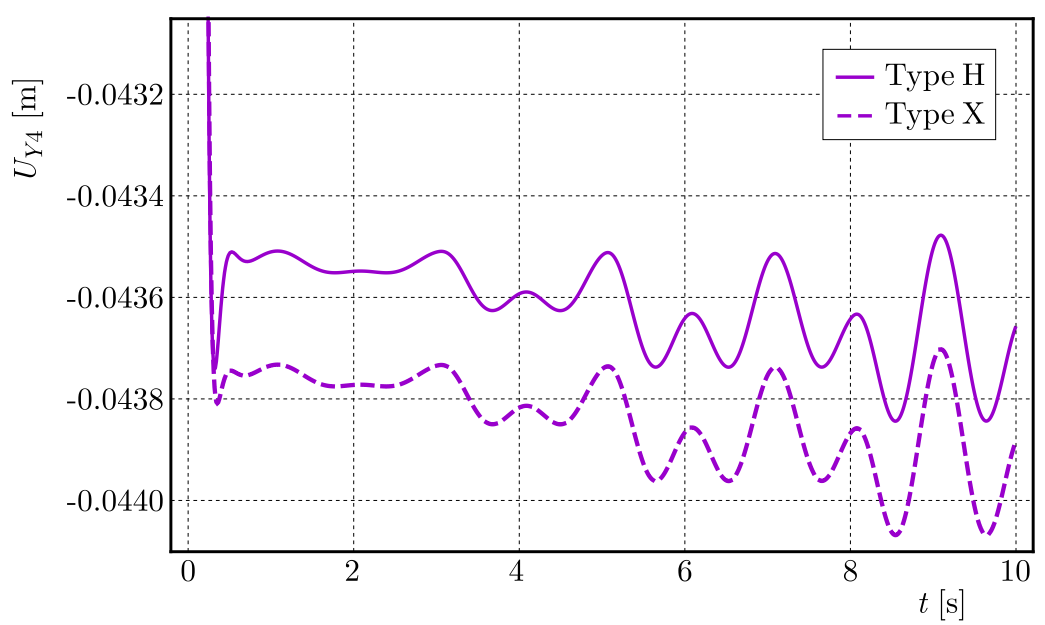

Fig. 16. Comparison of the dynamic displacement of node 4 for the two portals

\section{Conclusions}

The conclusions of a comparative dynamic analysis of the two $\mathrm{H}$ and $\mathrm{X}$ portal cranes are:

- It is possible to recognize the structural behaviour during free undamped vibrations and the dynamic response to identical excitations for the two types of portals.

- The first lowest (basic) vibration frequency of both portals is within the limits of [0.5-3.5] Hz, so the condition for small mass, or slenderness is fulfilled.

- The portal H is slightly more elastic than the portal X, which means that it has a slightly unfavorable dynamic stability as it has a lower first frequency of $7.66 \%$.

- Substations are provided that enable predicting the behaviour of the two types of portals due to the change of parameters (e.g., speed and acceleration of the crane, damping of the portal structure, stiffness of vertical springs,...).

- The positions of supports or vertical springs represent the most relevantly identified elements of both portal cranes for analyzing their dynamic behaviour.

- It is possible to analyse the behaviour of springs of the two portals for the identical excitation effect.

- The largest vertical displacement has a leg, i.e. a spring located on the opposite part of the portal in both axes in relation to the $45^{\circ}$ boom slewing in relation to rectilinear motion of the crane on the rails. 
- Increasing structural damping in the FE models of both portals leads to a decrease in the amplitudes of displacements.

- By comparing the displacements of the springs of the portals $\mathrm{H}$ and $\mathrm{X}$, it is concluded that they are very similar. The critical spring of the portal X has a higher vertical displacement by $0.45 \%$ than in the portal $\mathrm{H}$. In other words, the model $\mathrm{H}$ has a slightly higher dynamic stability for overturn than the model X;

- This paper leaves room for an extended dynamic analysis of the $\mathrm{H}$ and $\mathrm{X}$ portal cranes by advanced dynamic models, i.e. including more parameters.

\section{References}

1. Abdel-Rahman E.M., Nayfeh A.H, Masoud Z.N., 2003, Dynamics and control of cranes: a review, Journal of Vibration and Control, 9, 7, 863-909

2. Bathe K.J., 1996, Finite Element Procedures, Prentice-Hall, New Jersey

3. Chopra A.K., 2006, Dynamics of Structures, Prentice-Hall, New Jersey

4. Gustafsson T., 1996, On the design and implementation of a rotary crane controller, European Journal of Control, 2, 3, 166-175

5. Ito H., Hasegawa M., Irie T., Kato Y., 1985, Study on dynamic stability of a truck crane carrier: 2nd report, strict analysis of forward stability in load lowering motion, Bulletin of JSME, 28, 244, 2467-2473

6. Ju F., Chоо Y.S., Cui F.S., 2006, Dynamic response of tower crane induced by the pendulum motion of the payload, International Journal of Solids and Structures, 43, 376-389

7. Jerman B., Hribar A., 2013, Dynamics of the mathematical pendulum suspended from a moving mass, Technical Gazette, 20, 1, 59-64

8. LEE H.-H., 2004, A new motion-planning scheme for overhead cranes with high-speed hoisting, Journal of Dynamic Systems, Measurement and Control, 126, 2, 359-364

9. Maczyński A., Szczotka M., 2002, Comparison of models for dynamic analysis of a mobile telescopic crane, Journal of Theoretical and Applied Mechanics, 40, 4, 1051-1074

10. Petković Z., 1996, Metal Construction in Machine Building Industry 1 (in Serbian), Faculty of Mechanical Engineering Belgrade, Belgrade

11. Shaikh A.A., Kumar D.D., 2016, Lifting capacity enhancement of a crawler crane by improving stability, Journal of Theoretical and Applied Mechanics, 54, 1, 219-227

12. Spyrakos C., 1994, Finite Element Modeling in Engineering Practice, Algor ${ }^{\circledR}$, Pittsburgh

13. TowAREK Z., 1998, The dynamic stability of a crane standing on soil during the rotation of the boom, International Journal of Mechanical Sciences, 40, 6, 557-574

14. VAsiljević R., Gašić M., SAvković M., 2016, Parameters influencing the dynamic behaviour of the carrying structure of a type $\mathrm{H}$ portal crane, Journal of Mechanical Engineering, 62, 10, 591-602

15. Vasiljević R., Savković M., Bulatović R., 2013, The approaches to the mathematical-mechanical modeling supporting construction, IMK-14 - Research and Development in Heavy Machinery, 19, 1, EN29-38

16. Wu J.J., 2004, Dynamic responses of a three dimensional framework due to a moving carriage hoisting a swinging object, International Journal for Numerical Methods in Engineering, 59, 1679-1702

17. Wu J.J., Whittaker A.R., Cartmell M.P., 2000, The use of finite element techniques for calculating the dynamic response of structures to moving loads, Computers and Structures, $\mathbf{7 8}$, 789-799

Manuscript received September 14, 2018; accepted for print January 10, 2020 